\title{
Kopfschmerzen
}

\section{Neues zu Pathophysiologie und Therapie der Migräne}

\author{
V. Limmroth, H.-C. Diener, Neurologische Universitätsklinik Essen
}

NOTFALLMEDIZIN 2003; 29: 32-41

Migräne ist eine der häufigsten neurologischen Erkrankungen überhaupt. In den letzten zehn Jahren konnten beachtliche Fortschritte in der Therapie erzielt werden. Durch verbesserte bildgebende Verfahren, aber auch molekularbiologische und genetische Untersuchungen konnte das Verständnis zu Ätiologie und Pathophysiologie der Migräne deutlich verbessert werden. In der Akuttherapie stehen inzwischen neben den herkömmlichen Präparaten sieben Triptane mit unterschiedlichen pharmakologischen Profilen und Darreichungsformen zur Verfügung. Auch in der Prophylaxe kann inzwischen auf ein breites Repertoire unterschiedlicher Substanzen zurückgegriffen werden, sodass in vielen Fällen eine erfolgreiche Behandlung der Erkrankung durch ein individuell zugeschnittenes Therapieregime möglich ist.

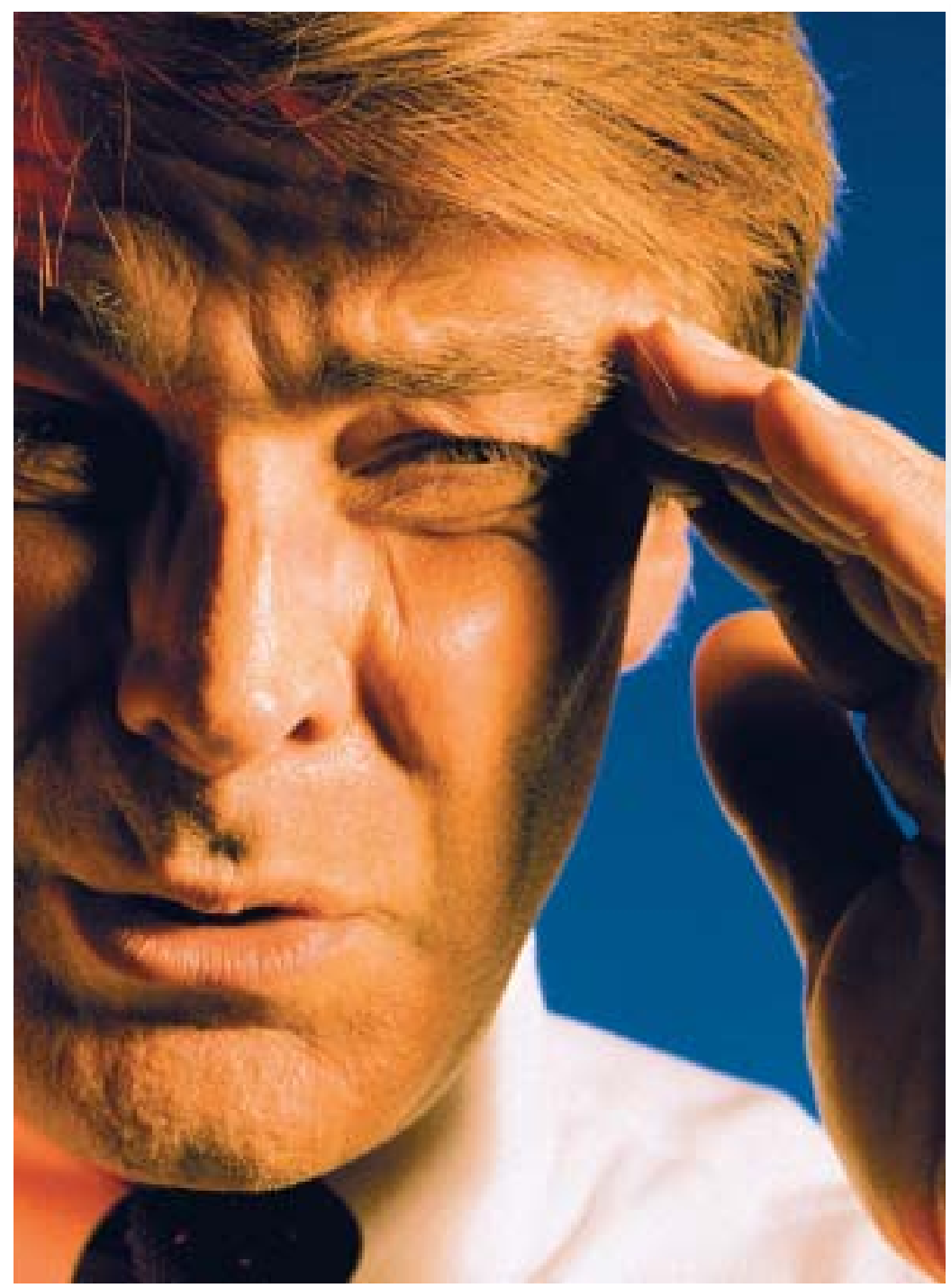

$\mathrm{M}$ igräne ist ein anfallsartig auftretender, nicht symptomatischer Kopfschmerz, der mit typischen autonomen Begleiterscheinungen wie Übelkeit, Erbrechen, Licht-, Geräusch- und Geruchsempfindlichkeit einhergeht. Mit einer weltweiten Prävalenz von $12-15 \%$ in der weiblichen und $5-7 \%$ in der männlichen Bevölkerung ist die Migräne eine der häufigsten neurologischen Erkrankungen überhaupt. Damit leben allein in der Bundesrepublik etwa acht Millionen Migräne-Patienten. Davon suchen nur zirka 20\% jemals wegen der Migräne einen Arzt auf und nur zirka $2 \%$ werden von einem Neurologen gesehen. Bei den meisten Patienten beginnt die Migräne während der Pubertät und hat ihren Häufigkeitsgipfel zwischen dem 35. und 40. Lebensjahr. Bei Kindern stehen vegetative Begleitsymptome wie abdominelle Schmerzen und Übelkeit im Vordergrund der Symptomatik.

Für eine Untergruppe der Migräne-Patienten konnte bereits ein genetischer Defekt auf Chromosom 1 oder 19 nachgewiesen werden. Bei diesen Patienten wird die alpha-Untereinheit eines $\mathrm{P} / \mathrm{Q}-\mathrm{Ca}^{++}$-Kanals falsch exprimiert. Aber auch für alle andere Formen wird inzwischen eine genetische Disposition im Sinne eines Ionenkanal-Defekts angenommen. Die einzelne Attacke wird dabei am ehesten durch den temporären Ausfall eines anti-nozizeptiven Zentrums im oberen Hirnstamm und die konsekutive Aktivierung serotonerger Bahnen initiiert. Die eigentliche Schmerzentstehung erfolgt dann durch die Aktivierung trigeminovaskulärer Bahnen und Freisetzung vasoaktiver Neuropeptide. Insbeson- 
dere die Entwicklung neuerer hochspezifischer Substanzen, wie der Serotonin (5-HT) $)_{1 \mathrm{~B} / \mathrm{D}}$-Agonisten (Triptane), hat neben den verbesserten bildgebungstechnischen Möglichkeiten sehr zum Verständnis der pathophysiologischen Abläufe beigetragen. Inzwischen stehen in Deutschland sieben verschiedene Triptane zur Therapie der Migräneattacke zur Verfügung: Almotriptan (Almogran ${ }^{\circledR}$ ), Eletriptan $\left(\operatorname{Relpax}^{\circledR}\right)$, Frovatriptan $\left(\right.$ Allegro $\left.^{\circledR}\right)$, Naratriptan (Naramig ${ }^{\circledR}$ ), Rizatriptan (Maxalt ${ }^{\circledR}$ ), Sumatriptan (Imigran ${ }^{\circledR}$ ) und Zolmitriptan (Ascotop ${ }^{\circledR}$ ). Im Folgenden soll ein kurzer Überblick über die notwendige Diagnostik, neue pathophysiologische Erkenntnisse und aktuelle Therapiemöglichkeiten in der Akutbehandlung sowie der Prophylaxe gegeben werden.

\section{Klinik und Diagnostik}

Klassischerweise kommt es meist in den Morgenstunden $\mathrm{zu}$ einem heftigen, pulsierenden und pochenden halbseitigen Kopfschmerz, der zum Teil aber auch den ganzen Kopf betrifft und mit Übelkeit, Erbrechen, Lichtscheu, Lärmempfindlichkeit und Geruchsüberempfindlichkeit einhergeht. Der Kopfschmerz nimmt typischerweise bei körperlicher Betätigung zu. Ein Migräneanfall kann zwischen vier und 72 Stunden dauern (im Durchschnitt 16 Stunden). Nicht wenige Patienten verspüren ferner so genannte Prodromalzeichen.

Diese Symptome treten bis zu 48 Stunden vor der eigentlichen Kopfschmerzphase auf und sind durch Heißhunger, Stimmungsschwankungen, euphorische Gefühle oder Polyurie gekennzeichnet. Typische Provokationsfaktoren sind Regelblutung, vorheriger Alkoholgenuss, Aufenthalt in verrauchten Räumen, Änderung des Schlaf-WachRhythmus, stressreiche Situationen oder Abfall des Koffein-Spiegels.

Je nach Klinik werden verschiedene Migräneformen unterschieden. Bei etwa 10-15\% der Patienten liegt eine Migräne mit Aura (früher klassische Migräne, Migraine accompagnée) vor. Hierbei kommt es vor oder selten unmittelbar zu Beginn der Kopfschmerzen zu neu- rologischen Reiz- oder Ausfallserscheinungen wie Gesichtsfelddefekten (Flimmerskotom), Wahrnehmung gezackter Figuren (Fortifikationen), halbseitigen Sensibilitätsstörungen, Paresen sowie Sprechoder Sprachstörungen. Die neurologischen Ausfälle entwickeln sich üblicherweise graduell über 5-20 Minuten und dauern nicht länger als 60 Minuten.

Eine Sonderform ist die Migräne mit prolongierter Aura, wobei die neurologischen Ausfälle bis zu maximal einer Woche anhalten und danach wieder völlig abklingen.

Die Diagnose kann ohne technische Zusatzuntersuchungen durch eine sorgfältige Anamnese (Ausschluss anderer symptomatischer Kopfschmerzen) und wenige spezifische Fragen gestellt werden:

- Ist der Kopfschmerz attackenartig oder dauerhaft?

- Dauer des Kopfschmerzes (Minuten, Stunden, Tage)

- Lokalisation des Kopfschmerzes, einseitig oder holocraniell

- Begleitsymptome: Übelkeit, Erbrechen, Licht-, Geräusch- und Geruchsempfindlichkeit.

Die klinisch-neurologische Untersuchung sollte unauffällig sein bzw. keine Hinweise auf symptomatische Kopfschmerzen bieten. Alle technischen Untersuchungen, insbesondere die kraniale Bildgebung, dienen lediglich dem Ausschluss symptomatischer Ursachen. Eine Zusatzuntersuchung, die die Diagnose einer Migräne bestätigen kann, gibt es nicht. Bei spezifischen Hinweisen in der Anamnese oder der klinisch-neurologischen Untersuchung auf einen symptomatischen Kopfschmerz müssen zum differenzialdiagnostischen Ausschluss gezielt die jeweiligen spezifischen Untersuchungen durchgeführt werden. Die wichtigsten Differenzialdiagnosen können Tabelle 1 entnommen werden.

\section{- Neues zur Pathophysiologie Neuroanatomie}

Aus anatomischen Studien und neurochirurgischen Berichten ist gut belegt, dass nur wenige intrakranielle Strukturen nozizeptive Impulse generieren können. Neuroanatomisch sind nur größere zerebrale Gefäße, meningeale und piale Gefäße, die großen venösen Sinus und die Dura von unmyelinisierten Fasern umgeben, die dem ersten ophtalmischen Abschnitt des Trigeminus-Ganglions sowie in der hinteren Schädelgrube der oberen zervikalen Hinterwurzel entspringen. Eine neuroanatomische Besonderheit, die erst Mitte der achtziger Jahre belegt wurde, ist, dass die Fasern, die aus Neuronen des Trigeminus-Ganglion entspringen, vasoaktive Neuropeptide wie Substanz P und Calcitonin gene related peptide (CGRP) enthalten. Diese Peptide sind alle starke Vasodilatoren und können nach Aktivierung der Fasern freigesetzt werden.

\section{Schmerzentstehung}

Erste Theorien zur Entstehung von Kopfschmerzen und auch der Aura-Symptomatik gingen von primär vaskulären Mechanismen aus. Dabei sollte eine initiale Vasokonstriktion zu einer dann schmerzhaften Vasodilatation kranialer Gefäße führen. Diese Theorien schienen vor allem durch die Wirksamkeit vasokonstriktiver Substanzen wie Ergotaminen gestützt zu werden. Vielfache Untersuchungen zu Blutflussveränderungen in größeren und kleineren kranialen Gefäßen konnten jedoch (außerhalb der Aura) keine signifikanten Flussänderungen zwischen der Attacke und dem schmerzfreien Intervall zeigen (7, 20, 34). Zwar konnte nach Verwendung von vasokonstriktiven Substanzen wie Ergotamin oder Sumatriptan eine Erhöhung der Blutflussgeschwindigkeit in intrazerebralen Gefäßen nachgewiesen werden, diese korrelierten jedoch nicht mit der Reduktion des Migräne-Kopfschmerzes (34). Auch tierexperimentelle Hinweise sprachen für periphere Entstehungsmechanismen, aber nicht im Sinne einer (reinen) Vasodilatation, sondern im Sinne einer „Neurogenen Entzündung“ der Dura, die verschiedene Komponenten einer aseptischen Entzündung umfasst $(44,45)$ mit Vasodilatation kleiner meningealer Gefäße, Plasmaextravasation in perivaskuläres Gewebe, Degranu- 
Aktuelles Wissen

\section{Tab. 1 Wichtige Differenzialdiagnosen der Migräne und notwendige Diagnostik}

\begin{tabular}{|c|c|c|c|}
\hline Differenzialdiagnose & Kopfschmerz und typische Anamnese & $\begin{array}{l}\text { Typisches Alter und } \\
\text { Patientenpopulation }\end{array}$ & $\begin{array}{l}\text { Notwendige weitere } \\
\text { Untersuchung }\end{array}$ \\
\hline Clusterkopfschmerzen & $\begin{array}{l}\text { Kopfschmerzdauer kürzer und intensiver als } \\
\text { bei Migräne (15-45 min), Rhinorrhö, } \\
\text { Ptosis, Lakrimation, keine Übelkeit oder } \\
\text { Erbrechen, bis zu } 3 \text { Attacken pro Tag }\end{array}$ & $\begin{array}{l}\text { Männer jüngeren und } \\
\text { mittleren Alters }\end{array}$ & $\begin{array}{l}\mathrm{CCT} / \mathrm{NMR} \text { initial zum } \\
\text { Ausschluss anderer Ursachen }\end{array}$ \\
\hline Trigeminus-Neuralgie & $\begin{array}{l}\text { Schmerzen wesentlich kürzer als bei } \\
\text { Migräne oder Clusterkopfschmerzen } \\
\text { (nur Sekunden!), Dutzende Attacken pro Tag }\end{array}$ & $\begin{array}{l}\text { Ältere Patienten } \\
\text { (über } 60 \text { Jahre) }\end{array}$ & $\begin{array}{l}\mathrm{CCT} / \mathrm{NMR} \text { initial zum } \\
\text { Ausschluss anderer Ursachen }\end{array}$ \\
\hline Subarachnoidalblutung (SAB) & $\begin{array}{l}\text { Heftigster nie gekannter, plötzlich } \\
\text { aufgetretener Kopfschmerz, aufgetreten } \\
\text { nach Anstrengung, Bewusstseinstrübung, } \\
\text { Meningismus }\end{array}$ & Jedes & $\begin{array}{l}\text { CCT, } \\
\text { Liquoruntersuchung: } \\
\text { blutiger Liquor. } \\
\text { Dann Angiographie: } \\
\text { Aneurysma? }\end{array}$ \\
\hline Intrazerebrale Blutung (ICB) & $\begin{array}{l}\text { Heftige Kopfschmerzen, fokal neurologische } \\
\text { Symptome, Krampfanfälle, vegetative Unruhe }\end{array}$ & Jedes & $\begin{array}{l}\text { CCT } \\
\text { Blutungsneigung, } \\
\text { Markumar }^{\circledR} \text { ?, } \\
\text { Hochdruckkrise? }\end{array}$ \\
\hline Sinusvenenthrombose & $\begin{array}{l}\text { Langsam über Wochen (bis zu } 8 \text { Wochen) } \\
\text { zunehmende Kopfschmerzen, therapiere- } \\
\text { fraktär, Krampfanfälle, Bewusstseins- } \\
\text { störungen }\end{array}$ & $\begin{array}{l}\text { Jedes, häufiger junge } \\
\text { Frauen mit Pille, Nikotin, } \\
\text { Schwangerschaft }\end{array}$ & NMR (CCT, wenn Spiral-CT) \\
\hline Arteriitis temporalis & $\begin{array}{l}\text { Über Tage bis wenige Wochen sich } \\
\text { entwickelnder heftiger einseitiger ganz- } \\
\text { tägiger KS u.U. Visusminderung, Schmerzen } \\
\text { beim Kauen (!) }\end{array}$ & $\begin{array}{l}\text { Über } 60 \text { Jahre nicht selten } \\
\text { mit Polymyalgia rheumatica }\end{array}$ & $\begin{array}{l}\text { BSG massiv erhöht, } \\
\text { Leukozytose, ggf. u.U. } \\
\text { Biopsie, sonst klinische } \\
\text { Diagnose }\end{array}$ \\
\hline Sinusitis & Schmerz dumpf, morgens Sekretabfluss & Jedes & Rö Nasennebenhöhlen, CCT \\
\hline Meningitis & $\begin{array}{l}\text { Insbesondere okzipitale und frontale } \\
\text { bilaterale Kopfschmerzen Fieber, } \\
\text { Meningismus (nicht immer stark), } \\
\text { Bewusstseinsänderungen }\end{array}$ & Jedes & Liquor entzündlich verändert \\
\hline Arterielle Hypertonie & Pochender Kopfschmerz, gerötetes Gesicht & Mittleres Alter & Blutdruck messen \\
\hline Arterielle Hypotonie & Macht keine Kopfschmerzen & $?$ & keine \\
\hline
\end{tabular}

lation von Mastzellen und Freisetzung von Entzündungsmediatoren wie Histamin, Serotonin, Prostaglandinen etc. (16). Diese meningealen oder pialen Vorgänge sind beim Menschen in vivo bisher jedoch nicht nachgewiesen worden.

Eindrucksvoll konnten dagegen Studien zur Peptidfreisetzung sowohl im Tierexperiment als auch bei Patienten in der Migräneattacke belegen, dass durch Aktivierung trigeminaler Fasern (Tierexperiment) und während der Migräneattacke die Konzentration von vasoaktiven Neuropeptiden wie Calcitonin gene related peptide (CGRP) im venösen
Blut deutlich ansteigt und nach Gabe von DHE (Dihydroergotamin) oder Sumatriptan wieder abfällt $(17,21)$. Daraus darf geschlossen werden, dass es während der Migräneattacke zu einer Aktivierung des Trigeminovaskulären Systems mit einer Freisetzung vasoaktiver Peptide kommt, die neben einer Vasodilatation auch weitere Vorgänge in Gang setzen. Auch bei anderen schmerzintensiven Kopfschmerzzuständen, wie dem Clusterkopfschmerz sowie bei der paroxysmalen Hemicranie, konnten inzwischen Erhöhungen von CGRP während der Schmerzphasen festgestellt werden, darüber hinaus - anders als bei der Migräne - begleitet von Erhöhungen anderer Peptide wie Vasointestinal Peptide (18), so dass in diesen Kopfschmerzerkrankungen im Gegensatz zur Migräne neben dem Trigeminovaskulären System auch andere (z.B. parasympathische oder symphatische) Fasern aktiviert sein könnten.

\section{Zentrale Mechanismen}

Die Mechanismen, die zur Aktivierung des Trigemino-vaskulären Systems während der Attacke führen, sind jedoch weniger klar. Werden die peripheren Endigungen der Fasern experimentell gereizt, kann 
eine verstärkte zelluläre Aktivierung in den trigeminalen Kernen des Hirnstamms, im Dorsalhorn und auf Höhe von $\mathrm{C} 1$ und $\mathrm{C} 2$ beobachtet werden (Untersuchungen an Katzen und Affen) (22). Interessanterweise färben sich die gleichen Neurone an, wenn der N. Occipitalis gereizt wird, was darauf hindeutet, dass dieselbe Gruppe von Neuronen Impulse sowohl von supraspinal als auch aus der Nackenregion erhält. Das wiederum erklärt nun gut, weshalb viele Patienten mit Migräneattacken über aus dem Nacken aufsteigende Schmerzen klagen: die Aktivierung der identischen Neurone projiziert Schmerzen in die Nackenregion. Folgt man (im Tierexperiment) dieser Faseraktivierung weiter, wird das Signal auf Fasern des quintothalamischen Trakts übertragen, der zum Thalamus führt. Innerhalb des Thalamus werden diese Impulse am ehesten durch die medialen Kerne des posterioren Komplexes und dem intralaminaren Thalamus geführt. Dass diese Mechanismen beim Menschen ebenfalls vorliegen, lieferte kürzlich eine wichtige kernspintomographische Studie, die die Aktivierung der zum Migränekopfschmerz kontralateralen Thalamusseite bei Patienten belegen konnte (1).

Umgekehrt führt die Reizung zentraler Strukturen, insbesondere eine bereits geringe Stimulation einzelner Hirnstammkerne zu nachhaltigen Änderungen des zerebralen Blutflusses. So kommt es nach der Stimulation des Nucleus coeruleus (v.a. noradrenerg) frequenzabhängig zu einer deutlichen Reduktion des Blutflusses durch einen alpha2-Adrenorezeptor vermittelten Mechanismus, der am Kortex am stärksten ausgeprägt ist. Die Stimulation des Nucleus raphe dorsalis (überwiegend serotonerg) führt hingegen $\mathrm{zu}$ einem deutlichen Anstieg des zerebralen Blutflusses. Passend dazu konnte beim Menschen während Migräneattacken eine deutliche Aktivierung dieser Hirnstammbereiche um das periaquäduktale Grau während der Migräneattacke, aber nicht bei anderen Schmerzbedingungen gesehen werden $(42,54)$. Die Tatsache, dass diese Bezirke nach Gabe von Sumatriptan zu- nächst aktiviert bleiben und erst im schmerzfreien Intervall wieder zur Ausgangsaktivität zurückkehren, erhärtet den Verdacht, dass diese Aktivierung kein Epiphänomen der Schmerzwahrnehmung ist, sondern eher in einem kausalen Zusammenhang mit der Generierung und Entstehung der Migräneattacke steht.

\section{Biologische und genetische Grundlagen der Migräne- entstehung}

Aus Zwillingsstudien aber auch anderen genetischen Untersuchungen ist bereits seit längerem eine erbliche Disposition der Migräne gut belegt (30). Ein entscheidender Fortschritt war der erstmals 1996 gelungene Nachweis eines Gendefektes bei Patienten mit einer familiär hemiplegischen Migräne (47). Patienten, die unter dieser relativ seltenen Form der Migräne leiden, zeigen im Rahmen ihrer Aura eine komplette Halbseitenlähmung für etwa 30-120 Minuten. Der Gendefekt konnte als fehlexprimierte alpha-Untereinheit eines $\mathrm{P} / \mathrm{Q}-\mathrm{Ca}^{++}$-Kanals analysiert werden. Inzwischen konnten weitere Arbeitsgruppen diese Daten bestätigen und ähnliche Defekte dieses $\mathrm{Ca}^{++}$-Kanals auf Chromosom 1 in anderen Familien, die unter der gleichen Symptomatik leiden, nachweisen. Einige der fehlexprimierten $\mathrm{Ca}^{++}$-Kanäle konnten inzwischen kloniert und neurophysiologisch untersucht werden. Hier zeigte sich, dass es unter bestimmten Bedingungen (Triggerfaktoren!) zu einer erhöhten Öffnungsfrequenz des defekten Kanals kommt, die dann zu einer (temporären) paralysierenden $\mathrm{Ca}^{++}$-Überladung der Zelle führt. Für diese Untergruppe der Migräne-Patienten besteht damit kein Zweifel an einer seltenen Ionenkanalerkrankung zu leiden. Die genetische Analyse dieser Familien war relativ einfach, eben weil diese Sonderform so selten ist. Für die allgemeine Migräne ist genau dieser Aspekt - die weite Verbreitung der Erkrankung - der Grund, weshalb die zugrundeliegenden genetischen Defekte so schwer einzugrenzen sind (klare Abgrenzung gesunder Kontrollgruppen nur schwer möglich). Analog zu dieser Untergruppe kann jedoch von einer komplexen und si- cherlich sehr heterogenen Ionenkanalstörung als biologische Ursache aller Migräneformen ausgegangen werden. Zweifellos gibt es Triggerfaktoren (Alkohol, sensorische Überflutung, Stress, Hormone), die die Schwelle einer Attackengenerierung beeinflussen und möglicherweise zum Auslösen von Attacken sogar notwendig sind, aber klar ist heute auch, dass das Konzept einer psychosomatischen Erkrankung für die Migräne Unsinn sein muss.

\section{Neues zur Pathophysiologie der Aura}

Gut 10-15\% aller Migräne-Patienten leiden neben ihren Kopfschmerzen auch an einer Aura meist visueller Art. Pathophysiologisch waren auch hier immer neurovaskuläre Mechanismen in Anlehnung an die von Leao bereits 1944 beobachtete Cortical spreading depression (CSD) vermutet worden. Danach kommt es nach einem Reiz des Kortex zu einer fortgesetzten Depolarisierung der Neurone, die eine Änderung des kortikalen Blutflusses nachsichzieht ohne die arterielle Versorgung des Kortex zu respektieren.

Auch hier konnten in den letzten Jahren neue bildgebende Techniken mit einer besseren zeitlichen Auflösung wesentliche Erkenntnisse zum Verständnis der Aura beitragen. Kernspintomographisch konnte die Hypoperfusion der Okzipitalrinde während der Aura bei Patienten nicht nur nachgewiesen (8), sondern auch quantifiziert werden (bis zu 50\% Reduktion). Entscheidend war dabei die (bereits diskutierte) Erkenntnis, dass die Reduktion des Blutflusses nicht im Sinne einer Ischämie (reduzierter Wassergehalt der Zelle), sondern im Sinne eines neuro-vaskulären Mechanismus erfolgte (Wassergehalt unverändert).

In einer eleganten Anordnung von Messungen gelang es der Bostoner Arbeitsgruppe von Mike Moskowitz kernspintomographisch ferner mit Hilfe des BOLD-Signals (blood oxygenation level-dependent) bei Patienten zu zeigen, dass das initiale Signal der Aura im extrastriatalen Kortex (Area V3A) entsteht und mit einer Geschwindigkeit von 3,5 +/- 
$1,1 \mathrm{~mm} / \mathrm{min}$ langsam über den okzipitalen Kortex nach frontal zieht (27). Darüber hinaus gelang es tierexperimentell kürzlich zu beweisen, dass meningeale Fasern durch CSD aktiviert werden können und damit die Freisetzung von vasoaktiven Neuropeptiden ausgelöst werden kann (4). Damit ist seit wenigen Jahren klar, dass CSD-artige Phänomene auch beim Menschen vorkommen können und Auren durch einen neuro-vaskulären Mechanismus entstehen. Grundlage der Auraentwicklung ist am ehesten ein spezifischer genetischer Defekt, der sich gut in Einklang mit einem $\mathrm{Ca}^{++}$Ionenkanaldefekt bringen ließe.

\section{Therapie der Migräne \\ Behandlung der akuten \\ Migräneattacke}

Die Deutsche Migräne- und Kopfschmerzgesellschaft sowie die Arzneimittelkommission der Deutschen
Ärzteschaft haben für die Behandlung der Migräne kürzlich Therapieempfehlungen erarbeitet. Die Therapie der akuten Attacke sollte sich danach an der Intensität des Kopfschmerzes und der Begleiterscheinungen orientieren. Als Basistherapie erfolgt bei leichten bis mittleren Attacken die kombinierte Gabe eines Analgetikums mit einem Antiemetikum. Dabei sollten jedoch zwei einfache, aber entscheidende Aspekte beachtet werden:

- die Dosierung muss ausreichen (z.B. mindestens $1000 \mathrm{mg}$ ASS oder 1000 mg Parazetamol)

- die Darreichungsform muss resorptionsfreundlich sein (Brausetabletten oder Granulat).

Die Gabe des Antiemetikums erfolgt zum einen um Übelkeit und Erbrechen zu bekämpfen, insbesondere jedoch um die Magen- und Darmtätigkeit anzuregen, die zu Be- ginn und während eines Migräneanfalls drastisch reduziert, für eine optimale Resorption von Analgetika jedoch unabdingbar ist. Dies erklärt, warum eine ganze Reihe von Arzneimitteln, die als Tabletten gegen die Schmerzen eingenommen werden, wenig wirksam sind: die Wirkstoffe werden nur sehr verzögert von Magen oder Darm in den Kreislauf aufgenommen. Als Antiemetikum kommen hier die verschreibungspflichtigen Substanzen Metoclopramid oder Domperidon zum Einsatz (siehe Tab. 2).

Bei schweren Migräneattacken sind bisher vor allem Mutterkornalkaloide eingesetzt worden. Da das Nebenwirkungsspektrum dieser Substanzgruppe jedoch dem der Triptane eindeutig unterlegen ist (insbesondere werden Übelkeit und Erbrechen bei vielen Patienten zunächst durch unselektive Bindung an dopaminerge Rezeptoren ver-

\section{Tab. 2 Medikamente zur Behandlung akuter Migräneattacken}

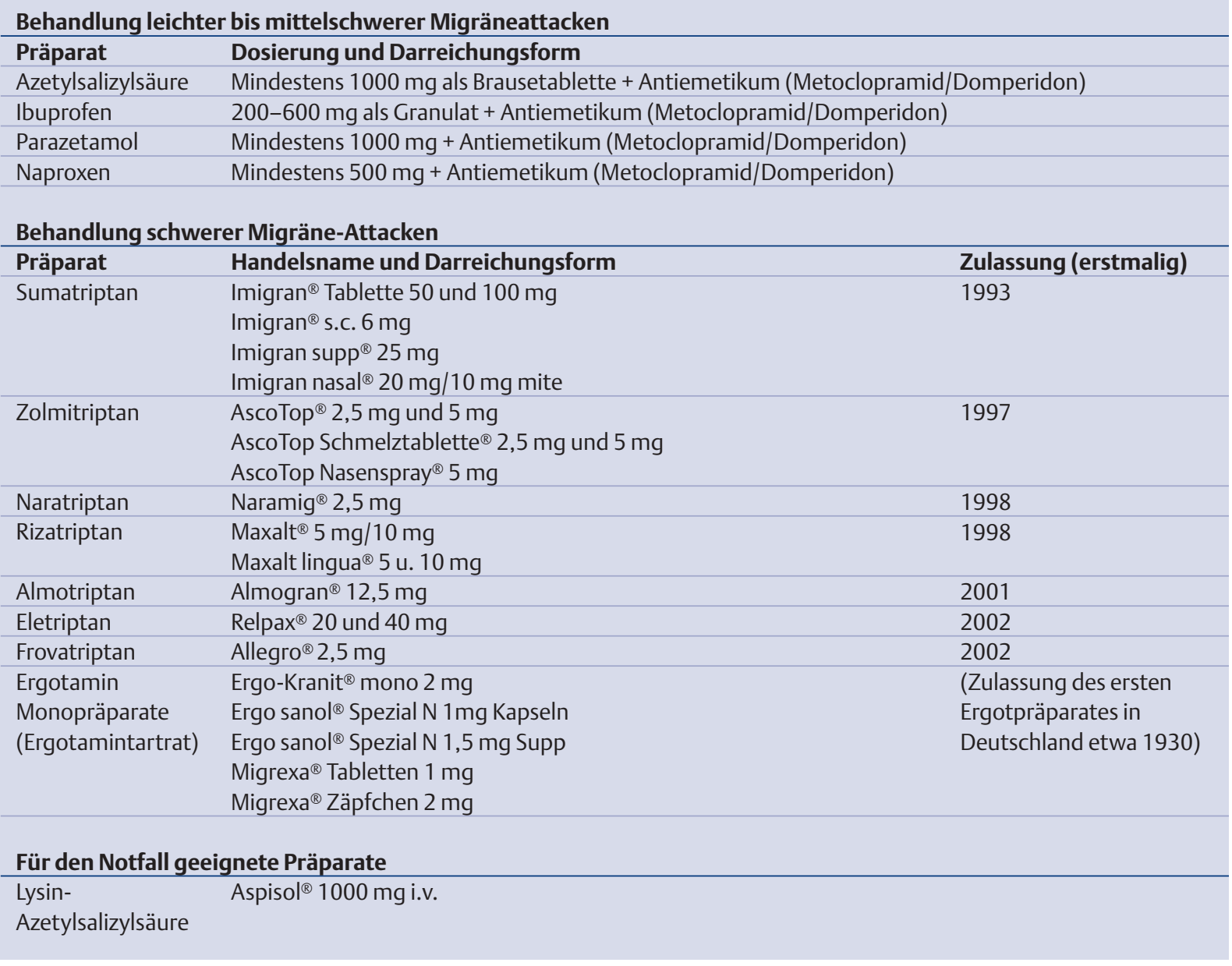


stärkt) und mehrere der neuen Triptane den Mutterkornalkaloiden bisher in allen klinischen Studien hinsichtlich ihrer Effektivität klar überlegen waren (10), können Mutterkornalkaloide heute nicht mehr als Mittel der ersten Wahl empfohlen werden. Der Einsatz von Mutterkornalkaloiden kommt daher nur noch als Ersatz infrage.

Neben der besseren Wirksamkeit liegt der Hauptvorteil der neuen Serotonin- (5- $\left.\mathrm{HT}_{1 \mathrm{~B} / \mathrm{D}^{-}}\right)$Agonisten gegenüber den älteren Ergotaminpräparaten vor allem in ihrer ausgeprägten antiemetischen Wirkung. Inzwischen stehen in Deutschland sieben $5 \mathrm{HT}_{1 \mathrm{~B} / \mathrm{D}}-$ Agonisten zur Verfügung (Details siehe 15). Für Patienten, die im Rahmen der Migräneattacke früh erbrechen und keine Tabletten einnehmen können, gibt es eine subkutane Form (Sumatriptan, $6 \mathrm{mg}$ ), die mithilfe eines Autoinjektors (ähnlich dem Diabetes-Pen) vom Patienten selbst appliziert werden kann. Die subkutane Gabe ist nach wie vor die am schnellsten wirksame Darreichungsform und führt bereits nach 15 Minuten bei etwa 25\% der Patienten zu einer Reduktion der Kopfschmerzen.

Für Patienten, die eine subkutane Injektion nicht wünschen oder vertragen, werden auch Zäpfchen (25 mg) und ein Nasenspray (20 mg) angeboten. Als weiteres Nasenspray wurde im Herbst 2002 Zolmitriptan auf den Markt gebracht. Erwähnenswert ist bei diesem Spray eine deutlich verbesserte Resorptionsfähigkeit durch die nasale Schleimhaut, sodass eine fast ähnliche Anflutzeit wie nach einer subkutanen Injektion erreicht wird. Zolmitriptan und Rizatriptan liegen ferner als Schmelztablette vor, die sich innerhalb von wenigen Sekunden auf der Zunge auflösen und nicht mit Wasser eingenommen werden müssen. Der Wirkungseintritt ist jedoch nicht schneller als bei den normalen Tabletten. Alle anderen Substanzen liegen ausschließlich in oralen Formulierungen als Tabletten vor.

Zwei der Substanzen wirken aufgrund ihrer Pharmakokinetik deutlich langsamer als alle anderen Triptane (Naratriptan und Frovatriptan). Diese Substanzen erreichen erst nach vier Stunden den Wirkungsgrad, den andere Substanzen bereits nach 60 bzw. 120 Minuten erzielen. Inzwischen sind einige der Triptane in Vergleichsstudien gegeneinander getestet worden. Dabei schlossen insbesondere Rizatriptan und Eletriptan besonders gut ab. Für die Details dieser Studien wird der Leser jedoch auf die Originalliteratur (z.B. 25) oder Metaanalysen (z.B. 19) verwiesen. Erwähnenswert sind ferner jüngere Studien mit neuen schnell anflutenden nicht-steroidalen Antirheumatika, die in ersten Studien den Wirkungsgrad des Sumatriptans erreichen (46). Diese Entwicklung wird durch weitere Studien bestätigt werden müssen.

Für den Notfall steht für Patienten, die schwerste Attacken haben, Kontraindikationen gegen Triptane oder Ergotamine zeigen oder bei denen andere Therapien bereits versagt haben, eine wasserlösliche Form der Azetylsalizylsäure, Lysin-Azetylsalizylsäure zur Verfügung $(12,36)$, die intravenös verabreicht werden kann und gut verträglich ist (einzige Kontraindikation: Asthma).

Alle $5-\mathrm{HT}_{1 \mathrm{~B} / \mathrm{D}}-$ Agonisten sind vasokonstriktorische Substanzen, die zwar wesentlich selektiver auf kraniale Gefäße wirken als Mutterkornalkaloide, die jedoch Patienten mit vaskulären Risikofaktoren nur unter Vorbehalt und Patienten mit koronarer Herzerkrankung unter keinen Umständen erhalten sollten (Kontraindikation).

\section{Die Zeit nach den Triptanen - Medikamente der Zukunft}

Nach inzwischen sieben zugelassenen Triptanen wird mit Donitriptan nun das vorläufig letzte Triptan in die klinische Entwicklung kommen $(29,38)$. Bis auf absehbare Zeit werden Triptane die Standard-Therapie für die Behandlung der akuten Migräneattacke bleiben. Trotz der erfreulichen Entwicklung der Triptane, die die Migränetherapie zweifellos ein großes Stück vorangebracht haben, können nicht alle Patienten mit den derzeit zur Verfügung stehenden Substanzen suffizient behandelt werden. In den meisten Therapiestudien bleiben bis zu 30\% aller Patienten unzureichend behandelt.
Die Entwicklung neuer Substanzen geht also weiter. Das ideale Migräne-Mittel der Zukunft sollte mindestens die Effektivität von Sumatriptan (als Standardpräparat) besitzen, aber keine vasokonstriktiven Eigenschaften haben. Erste Studien mit einem reinen $5-\mathrm{HT}_{1 \mathrm{~F}}$-Agonisten, der keine vasokonstriktiven Effekte hat, zeigten in einer Phase-II-Studie bereits signifikante Effekte in der Akuttherapie, die jedoch einer weiteren Bestätigung bedürfen (23). Mehrere Substanzen, die auf der Grundlage von Tiermodellen als geeignete Kandidaten erschienen, waren in klinischen Studien nicht wirksamer als Plazebo: Substanz-P-Antagonisten (13, 24), Endothelin-Antagonisten (41) und (reine) $5-\mathrm{HT}_{1 \mathrm{D}}$-Agonisten $(48,26)$. Als weitere Kandidaten zeigten sich kürzlich jedoch AdenosinA1-Agonisten und AMPA/ KA-Antagonisten, die in frühen Phase-II-Studien eine gute Wirkung in der Behandlung von Migräneattacken ohne kardio-vaskuläres Risikoprofil zeigten. Die wichtige Botschaft dieser Studien ist: auch nach den Triptanen wird es weitere Entwicklungen geben.

\section{Migräneprophylaxe}

Patienten mit häufigen Attacken laufen bei häufiger Einnahme von Analgetika, Ergotaminen oder auch neueren Substanzen wie Triptanen Gefahr, zusätzlich einen Medikamenten-induzierten Dauerkopfschmerz oder andere schädliche Nebenwirkungen zu erleiden. Diese Patienten müssen daher einer medikamentösen Dauerbehandlung zugeführt werden, die die Attackenfrequenz nachhaltig reduziert und auch auf Dauer gut verträglich ist.

Die Vorbeugung sollte jedoch bereits vor der Einleitung einer medikamentösen Prophylaxe mit spezifischen Änderungen im Verhaltensbereich beginnen, um unnötige Triggerfaktoren auszuschalten:

- Beibehaltung eines einheitlichen Schlaf-Wach-Rhythmus (auch am Wochenende)

- Einhaltung gleicher Koffeinmengen während der gesamten Woche (auch am Wochenende)

- Vermeiden übermäßigen Hungers

- Vermeiden von (übermäßigem) Alkohol und Nikotin 
Tab. 3 Medikamente zur prophylaktischen Behandlung der Migräne

\begin{tabular}{|c|c|c|}
\hline Substanz & Nebenwirkungen & Kontraindikationen \\
\hline Beta-Blocker & Müdigkeit, arterielle Hypotonie, & Asthma, AV-Block, Diabetes, \\
\hline Metoprolol (z.B. Beloc $\left.{ }^{\circledR}\right)(50-200$ mg) & Schlafstörungen (Albträume), & orthostatische Dysregulation \\
\hline Propranolol (z.B. Dociton $\left.{ }^{\circledR}\right)(40-160 \mathrm{mg})$ & $\begin{array}{l}\text { Schwindel, Broncheospasmus, } \\
\text { Bradycardie }\end{array}$ & \\
\hline Flunarizin (z.B. Sibelium $\left.{ }^{\circledR}\right)(5-10$ mg) & Müdigkeit, Gewichtszunahme (!) & $\begin{array}{l}\text { Dystonien, Schwangerschaft, Dystonien, } \\
\text { Parkinson? }\end{array}$ \\
\hline \multicolumn{3}{|l|}{ Mittel der zweiten Wahl } \\
\hline Substanz & Nebenwirkungen & Kontraindikation \\
\hline Valproinsäuren (z.B. Ergenyl ${ }^{\circledR}$ ) 500-900 mg & $\begin{array}{l}\text { Müdigkeit, Schwindel, Haarausfall, } \\
\text { Gewichtszunahme, Leberfunktions- } \\
\text { störungen }\end{array}$ & $\begin{array}{l}\text { Leberfunktionsstörungen, } \\
\text { - Schwangerschaft }\end{array}$ \\
\hline Naproxen (z.B. Aleve ${ }^{\circledR}$ ) 2 x 250 mg & Magenschmerzen & Ulkus, Blutungsneigung, Asthma \\
\hline Azetylsalizylsäure (z.B. Aspirin $\left.{ }^{\circledR}\right) 300$ mg & Magenschmerzen & Ulkus, Blutungsneigung, Asthma \\
\hline \multicolumn{3}{|l|}{ Mittel der dritten Wahl } \\
\hline Substanz & Nebenwirkungen & Kontraindikation \\
\hline Pizotifen (Sandomigran ${ }^{\circledR}$ ) 1-3 mg & $\begin{array}{l}\text { Müdigkeit, Gewichtszunahme, } \\
\text { Hunger, Mundtrockenheit, } \\
\text { Obstipation }\end{array}$ & Glaukom, Prostatahypertrophie, KHK \\
\hline Methysergid (Deseril $\left.{ }^{\circledR}\right) 2$ - $6 \mathrm{mg}$ & $\begin{array}{l}\text { Müdigkeit, Gewichtszunahme, } \\
\text { Hypertonus }\end{array}$ & Hypertonus, KHK \\
\hline Lisurid (Cuvalit $\left.{ }^{\circledR}\right) 3 \times 0,025$ mg & Müdigkeit, Übelkeit, Schwindel & Schwangerschaft, KHK, AVK \\
\hline
\end{tabular}

- Abbau von Stress (v.a. im Alltag)

- regelmäßige körperliche Betätigung, z.B. Jogging, Radfahren, Schwimmen und andere Ausdauersportarten.

Ziel der medikamentösen Prophylaxe ist es, Häufigkeit und Schwere der einzelnen Migräneanfälle zu vermindern sowie die Wirkung der Akutmedikation zu verbessern. Verlauf der Erkrankung sowie Erfolg beziehungsweise Misserfolg der Prophylaxe sollten anhand eines Kopfschmerztagebuches überprüft werden. Die Indikationen einer medikamentösen Prophylaxe sollten gestellt werden, wenn:

- mehr als drei Migräneanfälle im Monat auftreten

- mehr als eine Attacke pro Monat länger als 48 Stunden andauert

- mehr als eine Attacke pro Monat trotz jeglicher Akutmedikation therapierefraktär ist

- v.a. komplizierte Migräneattacken vorliegen (d.h. Migräneanfälle, bei denen die neurologischen Ausfallserscheinungen, wie Lähmungen oder Sehstörungen, länger als 24 Stunden anhalten)
- das Kopfschmerzleiden zu einer ausgeprägten beruflichen oder sozialen Beeinträchtigung und Gefahr für den Arbeitsplatz geführt hat.

Mittel der ersten Wahl sind auch weiterhin Betarezeptorenblocker. Am besten untersucht sind nach wie vor Metoprolol (z.B. Beloc ${ }^{\circledR}$ ) oder Propranolol (z.B. Dociton ${ }^{\circledR}$ ). Für beide Substanzen liegen über 50 klinische Studien zur prophylaktischen Wirkung bei Migräne vor. Auch andere Betablocker zeigten eine prophylaktische Wirkung, sind jedoch weniger gut untersucht. Bisoprolol (z.B. Concor $\left.{ }^{\circledR}\right)$, Atenolol (z.B. Tenormin $\left.^{\circledR}\right)$, Timolol (z.B. Dispatim ${ }^{\circledR}$ ) und Nadolol (Solgol ${ }^{\circledR}$ ) gehören zu der Gruppe der Betablocker, die wahrscheinlich eine prophylaktische Wirkung haben. Für Azebutol, Alprenolol, Oxprenolol und Pindolol konnte keine prophylaktische Wirkung belegt werden (37). Die Dosierungen sind Tabelle 3 zu entnehmen. Betablocker bieten sich insbesondere dann an, wenn Patienten unter Erkrankungen leiden, die ebenfalls mit Beta-Blockern behandelt werden können: Bluthochdruck, Tremor, in- nere Unruhe, Nervosität. Sie sind dagegen ungeeignet bei Patienten, die unter Depressionen leiden oder bei Männern mit Potenzproblemen.

Vergleichbar gut wirksam und damit noch Mittel der ersten Wahl ist der Kalzium-Antagonist Flunarizin (Sibelium ${ }^{\circledR}$ ), eine Substanz, die auch auf dopaminerge und serotonerge Rezeptoren wirkt (11). Da diese Substanz zu Beginn der Behandlung müde macht, sollte sie ausschließlich abends gegeben werden und ist insbesondere bei Patienten mit Schlafstörungen geeignet. Ungeeignet ist die Substanz hingegen bei Patienten mit Übergewicht (fast alle Patienten nehmen unter Flunarizin zu) oder mit depressiven Verstimmungen (Cave: Symptomzunahme). In wenigen Fällen wurde über extrapyramidale Nebenwirkungen berichtet, prädisponierte Patienten sollten es daher auch nicht erhalten.

Mittel der zweiten Wahl sind derzeit Valproinsäure und nichtsteroidale Antirheumatika (z.B. Naproxen oder ASS). Valproinsäure zeigte in einigen Vergleichsstudien eine den Betablockern vergleichbare Effektivität, die Substanz wird von 
einigen Autoren als Mittel der ersten Wahl betrachtet $(28,40,32)$. Bei den meisten Patienten sind jedoch niedrige Dosierungen um 600 mg ausreichend. Spezielle Nebenwirkungen können Gewichtszunahme, Tremor, Haarausfall (gering) und Leberschäden sein (selten). Da Valproinsäure im ersten Trimenon bei bis zu 5\% aller Fälle zu Neuralrohrdefekten führen kann, sollten Patientinnen im gebährfähigen Alter unter Valproinsäuretherapie einen antikonzeptionellen Schutz haben.

Erste Hinweise auf eine mögliche prophylaktische Wirkung von ASS kamen aus der PhysiciansHealth-Study (6), in der 22071 männliche Ärzte über einen Zeitraum von sechs Jahren regelmäßig 325 mg ASS oder Plazebo (jeden zweiten Tag) zur Prophylaxe gegen Herzinfarkt und Schlaganfall erhielten. In der Subgruppenanalyse zeigte sich eine Reduktion der Migränefrequenz von etwa $20 \%$ in der ASS-Gruppe. In weiteren Studien war ASS zwar jeweils besser wirksam als Plazebo, aber den Betablockern unterlegen $(14,35)$. Auch für Naproxen ist eine prophylaktische Wirkung gut dokumentiert (2, 3). NSARs eignen sich insbesondere für Patienten mit vaskulären Risikofaktoren, werden jedoch aufgrund der gastrointestinalen Nebenwirkungen nicht von allen Patienten toleriert.

Mittel der dritten Wahl sind die schon länger Verwendung findenden Serotoninantagonisten, wie Pizotifen (Sandomigran $^{\circledR}$, vom deutschen Markt genommen), Methysergid (Deseril ${ }^{\circledR}$ ) und Lisurid (Cuvalit $\left.{ }^{\circledR}\right)$, die bei Patienten aber wegen ihrer etwas stärkeren Nebenwirkungen (Müdigkeit, Gewichtszunahme) die Compliance beeinträchtigen können. Insbesondere Methysergid ist jedoch ein gut wirksames Prophylaktikum, führt aber zu erhöhtem Blutdruck, Müdigkeit, Gewichtszunahme und steht im Verdacht, Retroperitonealfibrosen zu verursachen. Es darf daher maximal sechs Monate hintereinander eingenommen werden.

Dihydroergotamin (z. B. Dihydergot $^{\circledR}$ ) ist in der Vorbeugung der Migräne wirksam, kann allerdings bei Patienten mit Migräne bei regel- mäßiger Einnahme zu einem Dauerkopfschmerz führen. Die Wirkung von Magnesium ist nach wie vor unklar, da die bisherigen Studien widersprüchlich sind. Eine Therapie mit Riboflavin (Vitamin $B_{6}$ ) kann aufgrund der einzunehmenden Mengen nicht empfohlen werden.

Entscheidend für die erfolgreiche Prophylaxe ist der Erhalt der Patienten-Compliance. Alle prophylaktisch verabreichten Substanzen sollten daher langsam eingeschlichen werden, um die typischen Nebenwirkungen gering zu halten. Die Wirkung ist selbst bei regelmäßiger Einnahme erst nach mehreren Wochen abschließend beurteilbar. Auch dies sollte dem Patienten mitgeteilt werden, um ein frühzeitiges Absetzen der Medikation zu verhindern.

\section{Neuere Antikonvulsiva in der Migräneprophylaxe}

Lamotrigin (Lamictal ${ }^{\circledR}$ ) ist in mehreren kleineren Studien in der Prophylaxe der Migräne untersucht worden (49), zeigte nur eine mit der Plazebo-Gruppe vergleichbare Wirkung und erscheint für MigräneProphylaxe damit ungeeignet. Zwei weitere Studien untersuchten ferner die spezifische Wirkung von Lamotrigin auf die Aura-Symptomatik. In beiden Studien konnte eine signifikante Reduktion der Aura-Frequenz sowie der Dauer beobachtet werden (9, 33). Damit könnte Lamotrigin eines der wenigen Medikamente sein, das zur Behandlung der Aura eingesetzt werden kann.

Gabapentin (Neurontin ${ }^{\circledR}$ ) ist bisher erst in einer doppelblinden Plazebo-kontrollierten Studien untersucht worden (39). Hier war die Substanz bei guter Verträglichkeit Plazebo klar überlegen und reduzierte in einer Dosis von $2400 \mathrm{mg}$ die Attackenfrequenz um etwa 50\%. Auch diese Daten bedürfen der Bestätigung. Nachteil sind jedoch die hohe Anzahl an Tabletten, die pro Tag eingenommen werden müssen, sowie der hohe Preis.

Für Topiramat (Topamax ${ }^{\circledR}$ ) liegt bisher eine veröffentlichte doppelblinde plazebo-kontrollierte Studie vor (50) sowie mehrere Einzelfallberichte. Danach könnte auch Topiramat eine prophylaktische Wirkung haben. Eine größere Multi-CenterStudie in Europa überprüft derzeit die Wirksamkeit. Problematisch bleiben jedoch die potenziellen Nebenwirkungen wie Müdigkeit und Konzentrationsstörungen. Topiramat ist jedoch die einzige Substanz, die im Gegensatz zu fast allen anderen Substanzen zu keiner Gewichtszunahme, sondern einer signifikanten Gewichtsabnahme führt. Topiramat könnte daher - Wirksamkeit vorausgesetzt - einen festen Platz bei der Behandlung von Patienten bekommen, die aufgrund ihres Übergewichts nicht mit Betablockern, Flunarizin oder Valproinsäure behandelt werden können. Zu Tiagabin, Levetiracetam und Zonisamid liegen bisher nur Einzelfallberichte vor, die jeweils von potenziellen Wirkungen berichteten. Hier müssen die ersten klinischen Studien abgewartet werden.

\section{Alternative Therapieformen bei der Migräne}

Als alternative Therapieformen werden (mit mäßigem Erfolg) Akupunktur und psychologische Behandlungsverfahren, wie Stressbewältigungstraining, die progressive Muskelrelaxation nach Jacobsen und sogenannte Biofeedback-Trainings durchgesetzt. Eine kürzlich geführte Metaanalyse aller Studien, die Akupunktur mit Scheinakupunktur in der Migräneprophylaxe verglichen hatten, fand jedoch lediglich marginale Unterschiede (43). Die Wirksamkeit der Akupunktur muss daher kritisch gesehen werden und bleibt mehr als fraglich. Biofeedback ist sehr aufwändig und wird nur in wenigen Zentren angeboten.

\section{Summary}

Migraine is one of the most frequently appearing neurological disorders. Within the last ten years, however, a great deal of progress could be made. Significant improvements in the field of neuroimaging, but as well through molecular and genetic studies provided new insights into etiology and pathophysiology of migraine. Aside the conventional NSAIDs, seven recently introduced triptans with different pharmacological properties and different route of administration are now available in most countries. Further- 
more, for the prophylactic treatment of migraine the physician is able to use a broad spectrum of different agents, and thus is - in most of the cases - able to provide a successful custom tailored therapeutic concept.

\section{Literatur}

1. Bahra A, Matharu M, Büchel C, and Frakowiak RS]. Brainstem activation specific to migraine headache. Lancet 2001; 357: 1016-7

2. Behan PO and Connelly K. Prophylaxis of migraine: a comparison between naproxen sodium and pizotifen. Headache 1986; 26, 237-239

3. Bellavance AJ and Meloche JP. Comparative study of naproxen sodium, pizotyline and placebo in migraine prophylaxis. Headache 1990; 30, 710-715

4. Bolay H, Reuter U, Dunn AK, Huang Z, Boas DA, Moskowitz MA. Intrinsic brain activity triggers trigeminal meningeal afferents in a migraine model. Nat Med 2002; 8 : 136-42

5. Brown EG, Endersby CA, Smith RN, Talbot JCC. The safety and tolerability of sumatriptan: an overview. Eur Neurol 1991; 31, 339-344

6. Buring JE, Peto R, and Hennekens $\mathrm{CH}$. Low-dose aspirin for migraine prophylaxis. JAMA 1990; 264, 1711-1713

7. Caekebeke JF, Ferrari MD, Zwetsloot CP, Jansen J, Saxena PR. Antimigraine drug sumatriptan increases blood flow velocity in large cerebral arteries during migraine attacks. Neurology 1992; 42: 1522-6

8. Cutrer FM, Sorensen AG, Weisskoff RM, Ostergaard L, Sanchez del Rio M, Lee E], Rosen BR, Moskowitz MA. Perfusion-weighted imaging defects during spontaneous migrainous aura. Ann Neurol 1998; 43: 25-31

9. D'Andrea G, Granella F, Cadaldini M, Manzoni GC. Effectiveness of lamotrigine in the prophylaxis of migraine with aura: an open pilot study. Cephalalgia 1999; 19:64-6 10. Diener HC, Jansen JP, Reches A, Pascual J, Pitei D, Steiner TJ. Efficacy, Tolerability and Safety of Oral Eletriptan and Ergotamine plus Caffeine (Cafergot((R)) in the Acute Treatment of Migraine: A Multicentre, Randomised, Double-Blind, Placebo-Controlled Comparison. Eur Neurol 2002; 47: 99-107 11 . Diener $C$. Flunarizine for migraine prophylaxis In: Diener HC (Ed.) Drug treatment of migraine and other headaches. Basel, Karger, 2000, 269-278

12. Diener HC, for the ASASUMAMIG Study Group. Efficacy and safety of intravenous acetylsalicylic acid lysinate compared to subcutaneous sumatriptan and parenteral placebo in the acute treatment of migraine. A double-blind, double-dummy, randomized, multicenter, parallel group study Cephalalgia 1999;19, 581-588

13. Diener HC, for the RPR100893-201 Migraine Study Group. Substance P antagonist RPR100893-201 is not effective in human migraine attacks 6th International Headache Research Seminar, Copenhagen 1995, 245-371

14. Diener HC, Hartung E, Chrubasik J, Evers S, Schoenen J, Latta G, Gendolla A. on behalf of the Study Group Acetylsalicylic acid in migraine prophylaxis: a double blind study in comparison with metoprolol In „8th Congress of the International Headache Society, Amsterdam" 1997

15. Diener HC, Limmroth V. Advances in the pharmacological treatment of migraine. Exp Opinion Invest Drugs 2001; 10: 1831-1845 16. Dimitriadou V, Buzzi MG, Moskowitz MA, Theoharides TC. Trigeminal sensory fiber stimulation induces morphological changes reflecting secretion in rat dura mater mast cells. Neuroscience 1991; 44: 97-112

17. Edvinsson L, and Goadsby PJ. Neuropeptides in migraine and cluster headache. Cephalalgia 1994; 14, 320-327

18. Edvinsson L, Goadsby PJ. Neuropeptides in headache. Eur J Neurol 1998; 5: 329-41

19. Ferrari MD, Roon KI, Lipton RB, Goadsby PJ. Oral triptans (serotonin 5-HT(1B/1D) agonists) in acute migraine treatment: a meta-analysis of 53 trials. Lancet 2001; 358: 1668-75

20. Friberg L, Olesen J, Iversen HK, Sperling B. Migraine pain associated with middle cerebral artery dilatation: reversal by sumatriptan. Lancet 1991; 338:13-7

21. Goadsby PJ, Edvinsson L. The trigeminovascular system and migraine: studies characterizing cerebravascular and neuropeptide changes seen in humans and cats. Ann Neurol 1993: 33, 48-56

22. Goadsby PJ, Zagami AS. Stimulation of the superior sagittal sinus increases metabolic activity and blood flow in certain regions of the brainstem and upper cervical spinal cord of the cat. Brain 1991; 114, 1001-1011

23. Goldstein DJ, Roon KI, Offen WW, Ramadan NM, Phebus LA, Johnson KW, Schaus JM, Ferrari MD. Selective seratonin $1 \mathrm{~F}$ (5HT(1F)) receptor agonist LY334370 for acute migraine: a randomised controlled trial. Lancet 2001;358:1230-4

24. Goldstein DJ, Wang O, Saper JR, Stoltz R, Silberstein ST, and Mathew NT. Ineffectiveness of neurokinin-1 antagonist in acute migraine: crossover study. Cephalalgia 1997; $17,785-790$

25. Goldstein J, Ryan R, Jiang K, Getson A, Norman B, Block G, Lines C, and the Rizatriptan Protocol 046 Study Group. Crossover comparison of rizatriptan $5 \mathrm{mg}$ and $10 \mathrm{mg}$ versus sumatriptan 25 and $50 \mathrm{mg}$ in migraine. Headache 1998; 38, 737-747

26. Gomez-Mancilla B, Cutler NR, Leibowitz MT, Spierings EL, Klapper JA, Diamond S, Goldstein J, Smith T, Couch JR, Fleishaker J, Azie N, Blunt DE. Safety and efficacy of PNU-142633, a selective 5-HT1D agonist, in patients with acute migraine. Cephalalgia 2001; $21: 727-32$

27. Hadjikhani N, Sanchez Del Rio M, Wu O, Schwartz D, Bakker D, Fischl B, Kwong KK, Cutrer FM, Rosen BR, Tootell RB, Sorensen AG, Moskowitz MA. Mechanisms of migraine aura revealed by functional MRI in human visual cortex. Proc Natl Acad Sci U S A 2001; 98:4687-92

28. Jensen R, Brinck T, and Olesen J. Sodium valproate has a prophylactic effect in migraine without aura: a triple-blind, placebo-controlled crossover study Neurology 1994; 44, 647-651

29. John GW, Pauwels PJ, Perez M, Halazy S, LeGrand B, Verscheure Y, Valentin IP, Palmier C, Wurch T, Chopin P, Marien M, Kleven MS, Koek W, Assie MB, Carilla-Durand E, Ta- rayre JP, Colpaert FCJ. F 11356, a novel 5-hydroxytryptamine (5-HT) derivative with potent, selective, and unique high intrinsic activity at $5-\mathrm{HT} 1 \mathrm{~B} / 1 \mathrm{D}$ receptors in models relevant to migraine. Pharmacol Exp Ther 1999; 290: 83-95

30. Joutel A, Bousser, M, Biousese, V, Labauge, P, Chabriat, H, Nibbio, A, Maciazek, J. Meyer, B, Bach, M, Weissenbach, J, Lathrop, $\mathrm{G} \mathrm{M}$, and Tournier-Lasserve E. A gene for familial hemiplegic migraine maps to chromosome 19 Nature Genetics 1993; 5, 40-45 31. Kaube H, Herzog J, Kaufer T, Dichgans $\mathrm{M}$, Diener $\mathrm{HC}$. Aura in some patients with $\mathrm{fa}$ milial hemiplegic migraine can be stopped by intranasal ketamine. Neurology 2000; 55: 139-41

32. Klapper, J, on behalf of the Divalproex Sodium in Migraine Prophylaxis Study Group Divalproex sodium in migraine prophylaxis: a dose-controlled study. Cephalalgia 1997: 17: 103-108

33. Lampl C, Buzath A, Klinger D, Neumann $\mathrm{K}$. Lamotrigine in the prophylactic treatment of migraine aura - a pilot study. Cephalalgia 1999; 19:58-63

34. Limmroth V, May A, Auerbach P, Wosnitza G, Eppe T, Diener HC. Changes of cerebral blood flow velocity after treatment with sumatriptan or placebo and its implication in the pathophysiology of migraine. J Neurol Sci 1996; 138, 60-65

35. Limmroth V, Katsarava Z, Diener HC. Acetylsalicylic acid in the treatment of migraine and other headache - a historical and current overview. Cephalalgia 1999; 19: 545-551

36. Limmroth V, May A, Diener HC. Lysineacetylsalicylic acid in acute migraine attacks. Eur Neurol 1999; 41, 88-93

37. Limmroth V, Michel MC. The Prevention of Migraine: a critical review with special emphasis on ß-blockers. Br J Clin Pharmacol 2001; 52: 237-243

38. Limmroth V, Katsarava Z, Liedert, B, Schubert K, Michel M, Diener HC. A new invivo rat model for CGRP release following the activation of the trigeminal vascular system. Pain 2001; 92, 101-106

39. Mathew NT, Rapoport A, Saper J, Magnus L, Klapper J, Ramadan N, Stacey B, Tepper $S$. Efficacy of gabapentin in migraine prophylaxis. Headache 2001; 4:119-28 40. Mathew NT, Saper JR, Silberstein SD, Rankin L, Markley HG, Solomon S, Rapoport AM, Silber Cl, and Deaton RL. Migraine prophylaxis with divalproex. Arch Neurol 1995; $52,281-286$

41. May A, Gijsman HJ, Wallnöfer A, Jones R, Diener HC, Ferrari MD Endothelin antagonist bosentan blocks neurogenic inflammation, but is not effective in aborting migraine attacks. Pain 1996; 67, 375-378 42. May A, Kaube H, Buchel C, Eichten C, Rijntjes M, Juptner M, Weiller C, Diener HC. Experimental cranial pain elicited by capsaicin: a PET study. Pain 1998; 74:61-6

43. Melchart $D$, Linde $K$, Fischer $P$, White $A$, Allais G, Vickers A, Berman B. Acupuncture for recurrent headaches: a systematic review of randomized controlled trials. Cephalalgia 1999; 19, 779-786

44. Moskowitz MA. Basic mechanisms in vascular headache. Neurologic Clinics 1990; 8, 801-815

45. Moskowitz MA. Neurogenic versus vascular mechanisms of sumatriptan and ergot alkaloids in migraine. TiPS 1992; 13, 307-311 
46. Myllyla VV, Havanka H, Herrala L, Kangasniemi P, Rautakorpi I, Turkka J, Vapaatalo $\mathrm{H}$, Eskerod O. Tolfenamic acid rapid release versus sumatriptan in the acute treatment of migraine: Comparable effect in a double-blind, randomized, controlled, parallel-group study. Headache 1998; 38 , 201-207

47. Ophoff RA, Terwindt GM, Vergouwe MN, van Eijk R, Oefner PJ, Hoffman SMG, Lamerdin JE, Mohrenweiser HW, Bulman DE, Ferrari M, Haan J, Lindhout D, van Ommen GB, Hofker MH, Ferrari MD, Frants RR. Familial hemiplegic migraine and episodic ataxia type- 2 are caused by mutations in the $\mathrm{Ca} 2+$ channel gene CACNL1A4. Cell 1997; 87, 543-552

48. Roon K I, Olesen J, Diener HC, Ellis P, Hettiarachchi J, Poole PH, Christianssen I, Kleinermans D, Kok JG, Ferrari MD. No acute antimigraine efficiacy of CP-122,288, a highly potent inhibitor of neurogenic inflammation: results of two randomized, double-blind, placebo-controlled clinical trials. Ann Neurol 2000; 47, 238-241

49. Steiner TJ, Findley LJ, Yuen AW. Lamotrigine versus placebo in the prophylaxis of $\mathrm{mi}$ graine with and without aura. Cephalalgia 1997; 17:109-12

50. Storey JR, Calder CS, Hart DE, Potter DL. Topiramate in migraine prevention: a double-blind, placebo-controlled study. Headache 2001; 41:968-75

51. Tfelt-Hansen P, Henry P, Mulder LJ, Schaeldewaert RG, Schoenen J, Chazot G. The effectiveness of combined oral lysine acetylsalicylate and metoclopramide compared with oral sumatriptan for migraine. Lancet 1995; 346, 923-926

52. Tfelt-Hansen $P$, Teall J, Rodriguez F, Giacovazzo M, Paz J, Malbecq W, Block GA, Reines SA, Visser WH. on behalf of the Rizatrip$\tan 030$ Study Group Oral rizatriptan versus oral sumatriptan: a direct comparative study in the acute treatment of migraine Headache 1998; 38, 748-755

53. The Diclofenac-K/Sumatriptan Migraine Study Group. Acute treatment of migraine attacks: efficacy and safety of a nonsteroidal antiinflammatory drug, diclofenac-potassium, in comparison to oral sumatriptan and placebo. Cephalalgia 1999; 19, 232-240

54. Weiller C, May A, Limmroth V, Jüptner M, Kaube H, van Schayck R, Coenen HH, Diener HC. Brain stem activation in spontaneous human migraine attacks. Nature Medicine 1995; 1, 658-660

\section{Anschrift des Verfassers}

PD Dr. Volker Limmroth

Leitender Oberarzt

Neurologische

Universitätsklinik Essen

Hufelandstr. 55

45122 Essen

\section{Schnelle Übersicht Medikamente in der Notfallmedizin}

\author{
M. Bastigkeit, Medikamente in der Notfallmedizin. \\ 5. durchgesehene Auflage, Verlagsgesellschaft \\ Stumpf \& Kossendey, Edewecht, Wien, 2001 \\ 6. Auflage erscheint 2003.
}

Z nehmende Forderungen an das fachliche Können der Rettungssanitäter (Stichwort Notkompetenz und in Österreich Notfallkompetenz) müssen in der Ausbildung und in der Literatur ihren Niederschlag finden. Dazu gehören auch umfassende Kenntnisse der Medikamente, mit denen auf dem Transport in ein Krankenhaus „weiterer Schaden vom Patienten abgewendet" werden kann. Neben dem pharmakologischen Wirkprofil sind Kenntnisse über Lagerung, Kontrolle des Bestandes auf dem RTW ebenso wichtig, wie z. B. die Mischbarkeit verschiedener Medikamente und deren Tropfgeschwindigkeit bei Infusionen. Es ist der Verdienst von Bastigkeit, ein Kompendium für die Jackentasche in übersichtlicher Form bereits in der 5 . Auflage vorgelegt zu haben. Zur schnellen Übersicht ist „der Bastigkeit“ gegliedert in mehrere Teile: allgemeine Pharmakologie der besprochenen Substanzen und spezielle Pharmakologie, in dem man rasch das Wichtigste auf einen Blick findet. Positiv hervorzuheben ist, dass im 3. Teil des Buches in dieser Liste auch die Aufschlüsselung der Mindesthaltbarkeit zu finden ist; das ist eine wertvolle Hilfe für die Bestückung der Rettungsfahrzeuge. Die Auswahl relevanter Daten für eine akute Situation ist bei Weitem die schwierigste Arbeit eines solchen Notfallkompendiums. Dies ist dem Autor - einem erfahrenen Fachdozenten im Rettungswesen - gelungen. Die Interpretation von Medikamentenwirkungen und die Beurteilung ihrer Wertigkeit bei speziellen, akuten Krankheitsbildern ist (bislang) immer noch subjektiv gefärbt. Zu selten gibt es ein Mittel der ersten Wahl, sondern der Rettungssanitäter muss Wirkungen und Nebenwirkungen etlicher unterschiedlicher Medikamente und Medikamentengruppen beherrschen. Noch übersichtlicher und einfacher in der Handhabung (bei Notfällen muss es oft genug schnell gehen) wäre das Buch, ließe der Autor noch mehr Unwichtiges weg. Bei der kritischen Durchsicht des Buches findet man auf S. 67 und 69 den Hinweis auf einen Ceiling-Effekt für Pethidin (Dolantin) und Morphin. Der Rezensent bezweifelt das und sieht darüber hinaus keinen Grund, warum die Zielgruppe des Buches dies wissen müsste. Wesentlicher erscheint als Kritikpunkt jedoch, dass der Spagat zwischen einem Lehrbuch der Pharmakologie und einem schnellen Nachschlagewerk gelingen muss, so dass der Autor immer wieder gezwungen ist, für die praktische Tätigkeit des Anwendenden nicht relevante pharmakologische Daten aus Gründen der Übersicht wegzulassen. In seiner handlichen und übersichtlichen Form ist das Buch „Medikamente in der Notfallmedizin" nicht nur dem Rettungssanitäter sondern auch zum schnellen Nachschlagen für den in der Notfallmedizin tätigen Arzt eine wertvolle Hilfe.

U. Rendenbach
Diese Arbeit erschien zuerst in psycho 2002 28: 251-259. Der Nachdruck erfolgt mit freundlicher Genehmigung der Autoren. 